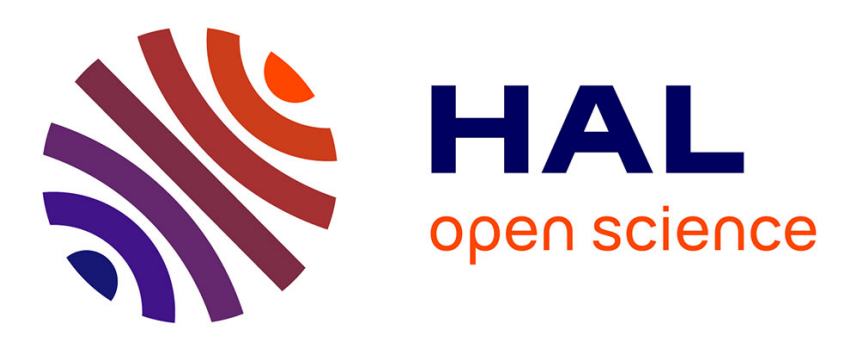

\title{
Estimation of SEA coupling loss factors using a dual formulation and FEM modal information, part II: numerical applications
}

\author{
Laurent Maxit, Jean-Louis Guyader
}

\section{- To cite this version:}

Laurent Maxit, Jean-Louis Guyader. Estimation of SEA coupling loss factors using a dual formulation and FEM modal information, part II: numerical applications. Journal of Sound and Vibration, 2001, 239 (5), pp.931-948. 10.1006/jsvi.2000.3193 . hal-01162187v2

\author{
HAL Id: hal-01162187 \\ https://hal.science/hal-01162187v2
}

Submitted on 14 Sep 2020

HAL is a multi-disciplinary open access archive for the deposit and dissemination of scientific research documents, whether they are published or not. The documents may come from teaching and research institutions in France or abroad, or from public or private research centers.
L'archive ouverte pluridisciplinaire HAL, est destinée au dépôt et à la diffusion de documents scientifiques de niveau recherche, publiés ou non, émanant des établissements d'enseignement et de recherche français ou étrangers, des laboratoires publics ou privés. 
Estimation of SEA coupling loss factors using a dual formulation and FEM modal information, part II: numerical applications

Reference: $\quad \mathrm{P} / 150 / 99$

Authors: $\quad$ Laurent MAXIT and Jean-Louis GUYADER

Address: $\quad$ INSA de Lyon (National Institute of Applied Sciences)

L.V.A. (Vibrations - Acoustic Laboratory)

Bat. 303

20, avenue Albert Einstein

69621 Villeurbanne Cedex

France

Phone: $\quad+33472436215$

+33472438712(fax)

Email: $\quad$ laurent.maxit@insa-lyon.fr

Headline: $\quad$ SEA COUPLING LOSS FACTORS

Total number of pages: $\quad 38$

Total number of figures: $\quad 12$

Total number of tables: $\quad 3$ 


\section{Summary:}

Numerical applications of the approach presented in the first of these two companion papers is proposed in the cases of coupled beams and coupled plates. Results are compared with other calculations. CLF calculation for coupled beams is achieved with analytical modal description. The influence of thickness ratio and damping about the quality of the prediction are presented and discussed. CLF determination with FEM modal description are then applied to the case of two thin plates coupled in an L shape. This case permit to achieve comparison with other calculation and will be representative of the applicability of the method to more complex structures as industrial ones. A very good agreement is noticed that validate the calculation of CLF with the present method. 


\section{INTRODUCTION}

In the first of these two companion papers, a theoretical method was presented to calculate the Coupling Loss Factor from subsystems modal information. By using Dual Modal Formulation and an appropriate subsystem mode definition, the expression which has be obtained (see (68) of part I) permits to determine CLF only from the knowledge of the modes of the uncoupled - subsystems and damping information. In the case of complex subsystems, the modal information can be calculated by Finite Element Method. The mode shapes are then described by nodal variables (displacements or forces) and the interaction modal work between couple of modes can be evaluated by considering the discretised model (see section 4, part I).

In this part, numerical applications of the approach is proposed to illustrate the method and to show validations with other calculations. Before going on to perform CLF calculations with FEM, one first example of coupled beams presents the CLF determination from the Dual Modal Formulation. In this section, modes are calculated analytically and results are compared with 'exact' calculation for different thickness ratios and different damping loss factors of the beams.

Validation of CLF calculation with FEM data are then presented. An example of L-shaped plates is considered, it is representative of the applicability of the method to more complex structures and allows us to compare to the results given by SEA inverse matrix technique with numerical experiments.

\section{A FIRST EXAMPLE}

\subsection{PRESENTATION}

This example is based on two Euler-Bernoulli beams coupled rigidly at one end as shown in Figure 1. The beams of rectangular section are simply supported at their ends, then the coupling are expressed by continuity conditions on the flexural moments and the angular rotations. 


\section{FIGURE 1}

$L_{\alpha}, b_{\alpha}, h_{\alpha}, E_{\alpha}, \rho_{\alpha}, v_{\alpha}$ are, respectively, length, width, thickness, Young's modulus, mass density, and Poisson's coefficient of beam $\alpha$. Then, the cross section area are $S_{\alpha}=b_{\alpha} h_{\alpha}$ and the surface inertia of beam $\alpha$ are $I_{\alpha}=12^{-1} b_{\alpha}\left(h_{\alpha}\right)^{3}$. In co-ordinate $x_{i}$, (i=1,2,3), the variables $W_{i}^{1}$ and $W_{i}^{2}$ $(i=1,2,3)$ represent the displacements and $\sigma_{i j}^{1}$ and $\sigma_{i j}^{2}$ are the stress tensors associated to beam 1 and beam 2, respectively. One defines a local co-ordinate system $(x, y, z)$ as represented Figure 1.

The Euler - Bernoulli assumptions on displacements and stresses for beam $\alpha$ are:

$$
\left\{\begin{array}{l}
W_{1}^{\alpha}(x, y, z, t)=-y \frac{\partial W_{y}^{\alpha}}{\partial x}(x, t), \\
W_{2}^{\alpha}(x, y, z, t)=W_{y}^{\alpha}(x, t), \quad \alpha=1,2 \\
W_{3}^{\alpha}(x, y, z, t)=0
\end{array}\right.
$$

where $W_{y}^{\alpha}(x, t)$ is the generalised transversal displacement of beam $\alpha$.

$$
\left\{\begin{array}{l}
\sigma_{11}^{\alpha}(x, y, z, t)=y \sigma_{x}^{\alpha}(x, t), \\
\sigma_{i j}^{\alpha}(x, y, z, t)=0,
\end{array} \quad \alpha=1,2\right.
$$

where $\sigma_{x}^{\alpha}(x, t)$ is the generalised normal stress of beam $\alpha$.

\subsection{ANALYTICAL DESCRIPTION}

One supposes the beams are constituted by the same material (aluminium) but one makes the hypothesis that beam 1 is thinner than beam 2 . The consequence is that beam 1 will vibrate rather like it is clamped at its coupling end and beam 2 will vibrate rather like it is simply supported at its 
coupling end. The subsystem definitions are then deduced naturally: the uncoupled - blocked subsystem for beam 1 and the uncoupled - free subsystem for beam 2. The boundary conditions for the extraction of subsystem modes are simply supported - clamped for beam 1, and simply supported - simply supported for beam 2 (see Figure 2).

\section{FIGURE 2}

Then, the subsystem 1 would have to be described by stress description, and the subsystem 2 would have to be described by displacement description. It is not necessary to re-develop the Dual Modal Formulation, because it is very simple to use directly the results of section 3 and the physical interpretation of the interaction modal work to determinate the modal coupling coefficients, $\gamma_{p q}^{12}$. In this example, the dual variables are the flexural moment and the angular rotation. The flexural moment, $\mathbf{M}_{f}^{1}$, is associated to beam 1 (stress description) and the angular rotation, $\theta_{z}^{2}$, is associated to beam 2 (displacement description). The interaction modal work exchanged by the $p^{\text {th }}$ mode of beam 1 and the $q^{\text {th }}$ mode of beam $2, \mathbf{W}_{p q}^{12}$, is expressed by the product of the flexural moment of the $p^{\text {th }}$ mode and the angular rotation of the $q^{\text {th }}$ mode at the coupling end:

$$
\mathbf{W}_{p q}^{12}=\tilde{\mathbf{M}}_{f}^{1 p}\left(L_{1}\right) \tilde{\theta}_{z}^{2 q}\left(L_{1}\right)
$$

where $\tilde{\mathbf{M}}_{f}^{1 p}\left(L_{1}\right)$ is the flexural moment of mode $p$ of beam 1 at the coupling end,

and $\tilde{\theta}_{z}^{2 q}\left(L_{1}\right)$ is the angular rotation of mode $q$ of beam 2 at the coupling end.

With Euler - Bernouilli theory, one has:

$$
\left\{\begin{array}{l}
\bullet \tilde{\mathbf{M}}_{f}^{1 p}(x)=I_{1} \tilde{\sigma}_{x}^{1 p}(x), \\
\text { where } \tilde{\sigma}_{x}^{1 p}(x) \text { is the stress shape of mode } p \text { of beam } 1, \\
\bullet \tilde{\theta}_{z}^{2 q}(x)=\frac{\partial \tilde{W}_{y}^{2 q}}{\partial x}(x), \\
\text { where } \tilde{W}_{y}^{2 q}(x) \text { is the displacement shape of mode } q \text { of beam } 2 .
\end{array}\right.
$$


To evaluate CLF from equation (68) of part I, one must calculate modes for each beam. The necessary information are for beam 1 natural frequencies, generalised mass and stress mode shapes at the coupling end, and for beam 2, natural frequencies, generalised mass and displacement mode shapes at the coupling end.

For beam 1, modal analysis is developed in Appendix A where two methods are presented to evaluate the stress mode shapes. Finally, the modal information can be expressed by (for $\left.\omega_{p}^{1} \neq 0\right)$ :

$$
\begin{aligned}
& \text { - } \omega_{p}^{1}=\sqrt{\frac{E_{1} I_{1}}{\rho_{1} S_{1}}}\left(k_{p}^{1}\right)^{2} \text { with } k_{p}^{1} / \quad \tan \left(k_{p}^{1} L_{1}\right)=\tanh \left(k_{p}^{1} L_{1}\right), \\
& \text { - } M_{p}^{1}=\rho_{1} S_{1} L_{1} \text {, } \\
& \text { - } \tilde{\sigma}_{x}^{1 p}(x)=\left(k_{p}^{1}\right)^{2} E_{1} I_{1}\left[\cosh \left(k_{p}^{1}\left(L_{1}-x\right)\right)+\cos \left(k_{p}^{1}\left(L_{1}-x\right)\right)\right. \\
& \left.-\frac{\cosh \left(k_{p}^{1} L_{1}\right)-\cos \left(k_{p}^{1} L_{1}\right)}{\sinh \left(k_{p}^{1} L_{1}\right)-\sin \left(k_{p}^{1} L_{1}\right)}\left(\sinh \left(k_{p}^{1}\left(L_{1}-x\right)\right)+\sin \left(k_{p}^{1}\left(L_{1}-x\right)\right)\right)\right]
\end{aligned}
$$

where $\omega_{p}^{1}$ is the natural angular frequency and $M_{p}^{1}$ the generalised mass of mode $p$.

For beam 2, the subsystem modal information is classical:

$$
\left\{\begin{array}{l}
\bullet \omega_{q}^{2}=\sqrt{\frac{E_{2} I_{2}}{\rho_{2} S_{2}}}\left(k_{q}^{2}\right)^{2} \text { with } k_{q}^{2}=\frac{q \pi}{L_{2}}, \\
\bullet M_{q}^{2}=\frac{\rho_{2} S_{2} L_{2}}{2} \\
\bullet \tilde{\mathrm{W}}_{y}^{2 q}(x)=\sin \left(k_{q}^{2}\left(x-L_{2}\right)\right),
\end{array}\right.
$$

where $\omega_{q}^{2}$ is the natural angular frequency and $M_{q}^{2}$ the generalised mass of mode $q$.

From equations $(4,5,8,11)$, one evaluate the expression of the interaction modal work (3):

$$
\mathbf{W}_{p q}^{12}=2\left(k_{p}^{1}\right)^{2} E_{1} I_{1} k_{q}^{2}
$$

One can notice that the modal informations necessary to evaluate the interaction modal work are only the mode shapes at the coupling end. 
The CLF must be evaluated by only taking into account the coupling between the resonant modes in the considered frequency bandwidth $\Delta \omega$. These resonant modes being chosen to resemble to physical modes of the global structure, it justifies that they describe the global behaviour of the structure in the frequency bandwidth. One note $N_{1}^{1}$ and $N_{2}^{1}\left(\operatorname{resp} . N_{1}^{2}\right.$ and $\left.N_{2}^{2}\right)$, the modal orders of resonant modes with the lowest and highest natural frequencies in $\Delta \omega$ for beam 1 (resp. beam 2). The number of resonant modes is $N_{1}=N_{2}^{1}-N_{1}^{1}+1$ for beam 1 and $N_{2}=N_{2}^{2}-N_{1}^{2}+1$ for beam 2 .

Then, with equation (68) of part I, (6,7), (10-12), the CLF is expressed by:

$$
\eta_{12}=\frac{1}{N_{1} \omega_{c}} \sum_{p=N_{1}^{1}}^{N_{2}^{1}} \sum_{q=N_{1}^{2}}^{N_{2}^{2}}\left\{\frac{8\left(k_{q}^{2}\right)^{2} E_{1} I_{1}}{L_{1} L_{2} \rho_{2} S_{2}}\left[\frac{\left[\Delta_{p}^{1}\left(\omega_{q}^{2}\right)^{2}+\Delta_{q}^{2}\left(\omega_{p}^{1}\right)^{2}\right]}{\left(\left(\omega_{p}^{1}\right)^{2}-\left(\omega_{q}^{2}\right)^{2}\right)^{2}+\left(\Delta_{p}^{1}+\Delta_{q}^{2}\right)\left(\Delta_{p}^{1}\left(\omega_{q}^{2}\right)^{2}+\Delta_{q}^{2}\left(\omega_{p}^{1}\right)^{2}\right)}\right]\right\},
$$

where $\Delta_{p}^{1}, \Delta_{q}^{2}$ are the modal damping bandwidths.

It can be noticed that the Damping Loss Factor can be attributed independently to each mode of each subsystem. However, in classical SEA, one global Damping Loss Factor is affected to each subsystem for all the modes of the frequency bandwidth. One assumes:

$$
\left\{\begin{array}{l}
\Delta_{p}^{1}=\omega_{p}^{1} \eta_{1}, \forall p \in\left[N_{1}^{1}, N_{2}^{1}\right], \\
\Delta_{q}^{2}=\omega_{q}^{2} \eta_{2}, \forall q \in\left[N_{1}^{2}, N_{2}^{2}\right],
\end{array}\right.
$$

where $\eta_{1}\left(\operatorname{resp} . \eta_{2}\right)$ is the D.L.F.of beam 1 (resp.beam 2).

With this assumption, (13) becomes:

$$
\eta_{12}=\frac{1}{N_{1} \omega_{c}} \sum_{p=N_{1}^{1}}^{N_{2}^{1}} \sum_{q=N_{1}^{2}}^{N_{2}^{2}}\left\{\frac{8\left(k_{q}^{2}\right)^{2} E_{1} I_{1}}{L_{1} L_{2} \rho_{2} S_{2}}\left[\frac{\left[\eta_{1} \omega_{p}^{1}\left(\omega_{q}^{2}\right)^{2}+\eta_{2} \omega_{q}^{2}\left(\omega_{p}^{1}\right)^{2}\right]}{\left(\left(\omega_{p}^{1}\right)^{2}-\left(\omega_{q}^{2}\right)^{2}\right)^{2}+\left(\eta_{1} \omega_{p}^{1}+\eta_{2} \omega_{q}^{2}\right)\left(\eta_{1} \omega_{p}^{1}\left(\omega_{q}^{2}\right)^{2}+\eta_{2} \omega_{q}^{2}\left(\omega_{p}^{1}\right)^{2}\right)}\right]\right\} .
$$

\subsection{NUMERICAL RESULTS}

The present approach is compared to the results yielded by the SEA matrix - inversion technique with numerical experiment (see reference [1]). Euler - Bernoulli theory and wave decomposition is used to simulate the numerical experiment. The 'rain on the roof' excitation on beam 1 is 
approximated by averaging beam energy over 20 points of excitation randomly distributed. The potential and kinetic energy obtained by the wave decomposition for a given angular frequency are spatial and frequency averaged. The Coupling Loss Factor, $\eta_{12}^{n e}$, is finally evaluated introducing beams energies in SEA relation when subsystem 2 is not excited:

$$
\eta_{12}^{n e}=\frac{\eta_{2}<E_{t}^{2}>}{<E_{t}^{1}>-\frac{n_{1}}{n_{2}}<E_{t}^{2}>}
$$

where $<E_{t}^{\alpha}>$ is the total energy of beam $\alpha$ obtained from numerical experiment, and $n_{\alpha}$ is the asymptoticmodal density of beam $\alpha$ given by analy tical expression :

$$
n_{\alpha}=L_{\alpha} \omega_{c}^{\frac{1}{2}}\left(\frac{\rho_{\alpha} S_{\alpha}}{E_{\alpha} I_{\alpha}}\right)^{\frac{1}{4}}
$$

where $\omega_{c}$ is the central angular radian frequency of the frequency bandwidth.

The results given by equation (15) are called 'CLF Dual Modal Formulation' or shortly CLF DMF, and the results given by the numerical experiment and equation (16) is called 'SEA matrix inversion'.

In what follows, the values $E_{\alpha}=7 \times 10^{+10} \mathrm{~N} / \mathrm{m}^{2}, v_{\alpha}=0.3, \rho_{\alpha}=2700 \mathrm{~kg} / \mathrm{m}^{3}, \alpha=1,2$ are always used.

\subsubsection{Intermodal Coupling Factors}

Let us first present in Figure 3 the Intermodal Coupling Factors, $\beta_{p q}^{12}$ as a function of couples of resonant modes of the two beams. One remembers that the CLF are obtained by summing up all these factors (see equation (15)).

$$
\beta_{p q}^{12}=\left[\frac{8\left(k_{q}^{2}\right)^{2} E_{1} I_{1}}{L_{1} L_{2} \rho_{2} S_{2}}\right]\left[\frac{\left[\eta_{1} \omega_{p}^{1}\left(\omega_{q}^{2}\right)^{2}+\eta_{2} \omega_{q}^{2}\left(\omega_{p}^{1}\right)^{2}\right]}{\left(\left(\omega_{p}^{1}\right)^{2}-\left(\omega_{q}^{2}\right)^{2}\right)^{2}+\left(\eta_{1} \omega_{p}^{1}+\eta_{2} \omega_{q}^{2}\right)\left(\eta_{1} \omega_{p}^{1}\left(\omega_{q}^{2}\right)^{2}+\eta_{2} \omega_{q}^{2}\left(\omega_{p}^{1}\right)^{2}\right)}\right] .
$$


Generally speaking, the Intermodal Coupling Factors vary considerably with the mode couple, this is due to two phenomena:

- the spatial coincidence of mode shapes at connection,

- the frequency coincidence (second bracket in (18)).

In this case of point connection, the frequency coincidence effect dominates and the Intermodal Coupling Factor is strong when $\omega_{p}^{1} \approx \omega_{q}^{2}$ (see Figure 3).

\section{FIGURE 3}

\subsubsection{Results for different thickness ratio of the two beams}

Figure 4 shows the CLF versus third octave band for four different beams thickness ratio. There is a good agreement between the results given by the approach developed in this paper and the results given by numerical experiment. This is true even when the thickness ratio is near one but a little difference (see Figure 4 (a)) is observed due to an increase of coupling strength. In this case, the difference of impedance between the two subsystems is not sufficient to assure that the resonant modes in the frequency band are completely able to represent the vibratory behaviour of the coupled subsystems.

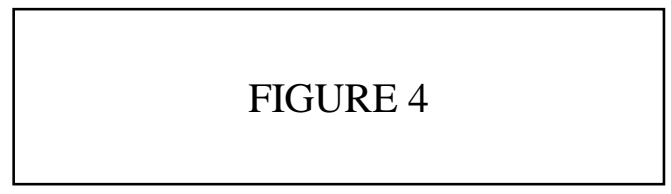

One extreme case is shown Figure 5 where the coupling of two identical beams is considered. In this situation, the choice of subsystem modes is arbitrary but in accordance with the Dual Modal Formulation, one subsystem should be blocked and the other should be free. The results given by the traditional wave approach (see expression (96) in [2]) are also plotted. As it was foreseeable, one can notice some differences between the results given by the two approaches and the 'exact' results. These differences are not so important (less than $5 \mathrm{~dB}$ ), then show that even in a severe 
coupling case, the results given by the present approach are not unsatisfactory. The improved wave theory given in reference [2] which do not require the many assumption of SEA can be more suitable for this system constituted by beams of equal thickness.

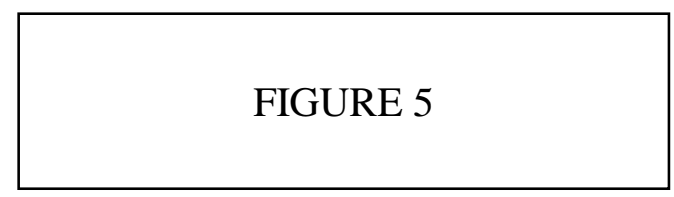

\subsubsection{Comparison results given by two opposite choices of modes}

Figure 6 shows the Coupling Loss Factor versus the thickness ratio $h_{1} / h_{2}$. Two calculations by the present approach have been made: The first one, which is plotted in solid line, considers blocked modes for beam 1 and free modes for beam 2, whereas, the second one, which is plotted with dash line, considers free modes for beam 1 and blocked modes for beam 2 . The modes are then reversed between the two calculations.

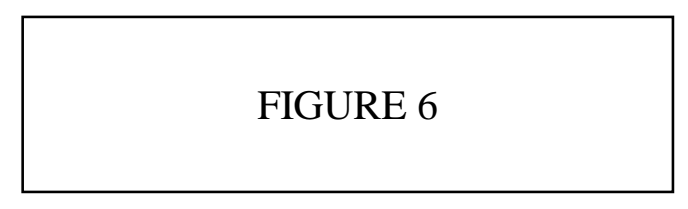

When the thickness ratio is less than one, the subsystem modes used by the first calculation are well suitable, whereas the second calculation gives poor results. Inversely, when the thickness ratio is bigger than one, the subsystem modes used by the second calculation are the most appropriates.

This allows us to emphasise that the choice of subsystem modes used to calculate the CLF by the present approach is not arbitrary. It should be achieved such that the subsystem modes are closest to the physical modes of the global structure. For example, when $h_{1} / h_{2}<1$, beam 1 is thinner than beam 2 and will vibrate rather like its is clamped at its coupling end whereas beam 2 will vibrate rather like it is simply supported at its coupling end. In this case, one can deduce naturally that beam 1 should be represented by the modes of the uncoupled - blocked subsystem whereas beam 2 should be represented by the modes of the uncoupled - free subsystem. 
Like at the previous section, one notices some slight errors for beams of equal thickness whatever the choice of modes (which become arbitrary in this case).

\subsubsection{Results for different damping}

Figure 7 shows the Coupling Loss Factor versus third-octave band when beams Damping Loss Factor are modified. In Figure $7(a-b-c)$ the DLF are the same for the two beams. A very good agreement can be seen between both method of calculation. In the case of low damping, the difference is larger (see Figure 7 ( c )). In such a situation, one can use a more detailed approach (SmEdA) presented in references [3], [4] which permits to obtain better results. This method allows us to take into account modal energy distribution, and the calculations from the Intermodal Coupling Factors accurate energy results when modal overlap is low.

In Figure 7 (d), the case of different Damping Loss Factors for the two beams is investigated. As previously, the presented method agrees well with the classical calculation with (16).

It can be noticed from the results given here that the CLF values depend slightly on damping which justify that the classical travelling wave approach gives results independent of damping. However, for very low damping, the damping dependence of CLF is more important (see [2],[5]) and in this case, SmEdA approach can be necessary (see [3], [4]).

The present approach agrees with the numerical experiment method but present the major advantages of giving CLF without solving equations, just making analogy with basic SEA modelisation. The computation time is thus very short.

FIGURE 7 


\section{APPLICATION OF CLF DETERMINATION WITH FEM DATA}

To demonstrate the validity of the approach, the simple case of two coupled homogeneous plates in an $\mathrm{L}$ shape as shown in Figure 8 is considered.

For Finite Elements point of view, the matrix form of the equation of motion is the same for complicated and simple structures. So, in principle, the treated problem will be representative of the applicability of the method to more complicated cases. In addition, this case allows us to make a comparison with another calculation.

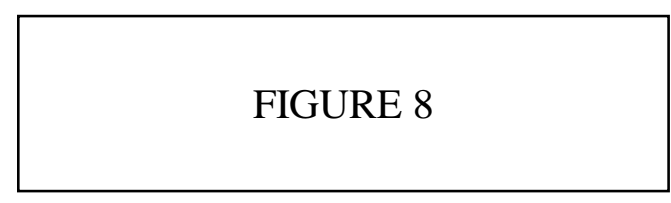

Thin plate theory is assumed and only bending motion is considered. All the non coupled edges of each plate are supposed simply supported. The parameter definitions and their values are presented table 1.

\section{TABLE 1}

Plate 1 is thinner than plate 2 , then it must be described by modes of the uncoupled - blocked subsystem, and plate 2 must be described by modes of the uncoupled - free subsystem. Thus, for subsystem definitions, plate 1 is clamped on the coupling edge and simply supported on the others edges. Plate 2 is simply supported on all the edge.

\subsection{FINITE ELEMENT MODEL}

The Finite Element Model of each subsystem are generated and analysed by UAI/NASTRAN v11.8 package and by using the QUAD4 Element. The numbers of nodes of the regular meshes are chosen in order to have a minimum of ten elements by natural wave length at $3000 \mathrm{~Hz}$. Then, for plate 1 there are 100 nodes along each edges, and for plate 2 there are 100 nodes for the smallest 
edge in order to have coincidence meshing and 130 nodes for the other one. Appropriate boundary conditions and Lanczos method are used to calculate the modes between $1 \mathrm{~Hz}$ and $3000 \mathrm{~Hz}$ for each plate. The generalized masses are normalised to one. The NASTRAN output data for each mode are the natural frequency, the 'SINGLE POINT CONSTRAINT FORCE' for plate 1, and the 'DISPLACEMENT' for plate 2. The present approach requiring only the mode shapes on the coupling boundary, it is possible to select only the nodes on the coupling boundary as a set of output (coupling node set). That allows us to limit the size of the NASTRAN output files. For example, for plate 1 , this output set is composed of the nodes, which are fixed, and the shape output data are described by the 'SINGLE POINT CONSTRAINT FORCE'. The output necessary to the CLF determination (see table 2) are extracted from the NASTRAN output files and are analysed by a MATLAB program.

\section{TABLE 2}

One uses equation (80) of part I to determine the interaction modal work from nodal components of mode shapes. In this case of flexural plates coupling, only the $5^{\text {th }}$ force nodal component for plate 1 (y-component of bending moment) and the $5^{\text {th }}$ displacement nodal component for plate $2(y$ component of angular rotation) are not null for the nodes of the coupling node set. Then, the interaction modal work between mode $p$ of plate 1 and mode $q$ of plate 2 can be reduced to:

$$
W_{p q}^{12}=\sum_{i \in\left\{\begin{array}{c}
\text { Coupling } \\
\text { node set }\}
\end{array}\right.} \tilde{f}_{p 5}^{1 i} \tilde{u}_{q 5}^{2 i},
$$

where $\tilde{f}_{p 5}^{1 i}$ is the $5^{\text {th }}$ nodal component force of node $i$ representing the $y$-component bending moment of the $p^{\text {th }}$ mode of plate 1 , and $\tilde{u}_{q 5}^{2 i}$ is the $5^{\text {th }}$ nodal component displacement of node $i$ representing the $y$-component angular rotation of the $q^{\text {th }}$ mode of plate 2 . 


\subsection{MODAL DENSITY COMPARISON}

\section{TABLE 3}

To check the validity of the FEM models in the frequency band of interest, it is possible to count the natural frequencies (see table 3), then determine the modal density for each frequency band and finally compare it with the asymptotic value $n_{\alpha}$ given by:

$$
n_{\alpha}=\frac{\sqrt{3} A_{\alpha} B_{\alpha}}{H_{\alpha} \sqrt{\frac{E_{\alpha}}{\rho_{\alpha}\left(1-v_{\alpha}\right)}}}, \alpha=1,2 . \quad(\operatorname{modes} / \mathrm{Hz})
$$

This is presented in Figure 9 where the modal density ratio between the two subsystems is plotted versus third octave band. One can conclude that the FEM modelisation is well representative of the vibration behaviour of the plates in the frequency range of interest. It is well known that the eigen-frequencies and the mode shapes of the Finite Element Model may be shifted to the real eigen-frequencies and mode shapes at 'high frequency' even in the case of a correct element model. However, as explained in reference [6], these numerical errors seem to be reduced by averaging the data.

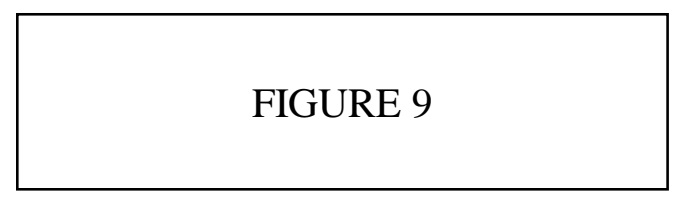

\subsection{COUPLING LOSS FACTOR COMPARISON}

From the expression of the interaction modal work for discretised system (19) and with the FEM data described in section 3.1, one can evaluate the Coupling Loss Factor (with (68) of part I). One calls this result the CLF-DMF/FEM.

In the same manner that in section 2, the present approach with FEM data is compared to the results yielded by the SEA matrix - inversion technique with numerical experiment. The 'exact' 
results of the numerical experiment are obtained from the Dual Modal Formulation with analytical modal description. A comparison with the general dynamic stiffness method allows one to check the convergence of the modal series. The 'rain on the roof' excitation on plate 2 is approximated by averaging plate energy over 15 points of excitation randomly distributed. Energies are spatial and frequency averaged. CLF is finally calculated introducing plate energies in SEA relation when subsystem 1 is not excited:

$$
\eta_{12}^{n e}=\frac{\eta_{1}<E_{t}^{1}>}{\frac{n_{1}}{n_{2}}<E_{t}^{2}>-<E_{t}^{1}>}
$$

where $<E_{t}^{\alpha}>$ is the total energy of plate $\alpha$ obtained from numerical experiment, and $n_{\alpha}$ is the modal density of plate $\alpha$ given by (20).

In Figure 10, one presents a comparison of three techniques of CLF calculation: The two techniques previously described and the classical technique derived from the travelling wave approach (see equations (20-23) in reference [7]).

One shows a good agreement between the three results in high frequency. Below 800 Hertz, the travelling wave approach which considers semi - infinite plates gives bad results for some third octave band whereas the present approach takes correctly into account the coupling between the two plates.

\section{FIGURE 10}

Figure 11 present a SEA calculation using the CLF-DMF/FEM when plate 2 is excited. This calculation is compared to an exact calculation (used previously in the SEA matrix - inversion technique). A very good agreement can be noticed that definitely validate the calculation of CLF with the present method.

FIGURE 11 
The important variations of energy ratios for the frequency bands below $1000 \mathrm{~Hz}$ can be explained by the fact that there is few resonant modes which participate to the coupling in these bands. Indeed, figure 12 shows the Intermodal Coupling Factors for the third octave band centred on $800 \mathrm{~Hz}$. Although plate 1 has 20 resonant modes in the frequency band and plate 2 has 12 resonant modes, one notices that only two couples of modes participate mainly to the coupling of plates. Contrary to beam coupling, the spatial coincidence has an important effect which leads some couple of modes to be uncoupled.

\section{FIGURE 12}

Like for beams of equal section (see section 2.3.2), the coupling of plates of equal thickness is a limit case of application of the present approach. Some slight errors will be made by this approach because the resonant subsystem modes which are considered could not fully represent the behaviour of the coupled plates. In this case of two edges-coupled rectangular plates of equal thickness, the calculations proposed by Mace in [8] can be more appropriate because they do not require the many SEA assumption.

\section{CONCLUSIONS}

Beam examples have permitted to verify the validity of the CLF determination with the Dual Modal Formulation and basic SEA relation for power flow in oscillators having gyroscopic coupling. It is shown that results are good when SEA assumptions are respected.

On another hand, one has seen that the present approach can be used in connection with FEM. Application of the SEA-CLF/FEM technique gives good estimates of vibrational energy ratios between two coupled plates for the third octave band between $315 \mathrm{~Hz}$ and $2500 \mathrm{~Hz}$. Although eigenfrequency shift occur in FEM calculation at high frequency, one gets good results for CLF. This is due to the averaging over several couples of modes participating to the transfer. The simple 
plate example has been chosen to allow us a comparison. The application to more complicated structures is straightforward because the technique is based about any general Finite Element Model. It can be noticed that heterogeneous substructures having three dimensional vibration motions can be treated without difficulty by CLF-DMF/FEM technique.

When a subsystem has a low number of modes in the frequency band, SEA can give poor estimate of energy transfer, because some assumptions used in the method are not realistic (equipartition in particular). The approach described in these two companion papers is a starting point of an extension of SEA to non modal energy equipartition in subsystems. This SEA extension called SmEdA (Statistical modal Energy distribution Analysis) is briefly presented in reference [3] and will be developed in another paper. The purpose of SmEdA is to extend the validity of SEA to system of low modal density.

\section{ACKNOWLEDGMENT}

The authors are grateful for the interest and financial support of DGA/DSP-CNRS (Direction des Systèmes de forces et de la Prospective) and CTSN Toulon (Centre Technique des Systèmes Navals), without which this work would not have been done. 


\section{REFERENCES}

[1] C. SIMMONS and C. FREDO 1990, Vibration analysis of coupled stiffened plates using the finite element method. In: internoise'90. International Conference on Noise Control Engineering, Gothenburg, Sweden, August 1990.

[2] B.R. MACE 1993 Journal of Sound and Vibration 166(3), 429-461. The statistical energy analysis of two continuous one-dimensional subsystems.

[3] L. MAXIT and JL. GUYADER 1999 Statistical modal Energy distribution Analysis (SmEdA). In $6^{\text {th }}$ International Congress on Sound and Vibration, Copenhagen, Denmark, 5-8 July 1999.

[4] L. MAXIT 2000 Ph.D. Thesis, Institut National des Sciences Appliquées de Lyon, France. Extension et reformulation du modèle SEA par la prise en compte de la répartition des énergies modales.

[5] F.F. YAP and J. WOODHOUSE 1996, Journal of Sound and Vibration 197(3), 351-371. Investigation of damping effects on statistical energy analysis of coupled structures.

[6] C. SIMMONS 1991 Journal of Sound and Vibration 144(2), 215-227. Structure-borne sound transmission through plate junctions and estimates of SEA coupling loss factors using finite element method.

[7] D.A. BIES and S. HAMID 1980 Journal of Sound and Vibration 70(2), 187-204. In situ determination of coupling loss factors by the power injection method.

[8] E.C.N. WESTER and B.R. MACE 1996 Journal of Sound and Vibration 193(4), 793822. Statistical energy analysis of two edge-coupled rectangular plates: ensemble averages.

[9] O. BERNASCONI and D.J. EWINS 1989 Journal of Modal Analysis 4(2), 68-76. Modal strain/stress fields. 


\section{APPENDIX A: MODAL ANALYSIS OF UNCOUPLED - BLOCKED BEAM 1}

Without applied forces and displacement, the free motion of the uncoupled - blocked subsystem

- beam 1 - respect the following equations:

$$
\begin{gathered}
\left.\rho_{1} S_{1} \frac{\partial^{2} W_{y}^{1}}{\partial t^{2}}(x, t)+I_{1} \frac{\partial^{2} \sigma_{x}^{1}}{\partial x^{2}}(x, t)=0, \quad \forall x \in\right] 0, L_{1}[, \\
\left.-E_{1} I_{1} \frac{\partial^{2} W_{y}^{1}}{\partial x^{2}}(x, t)+I_{1} \sigma_{x}^{1}(x, t)=0, \quad \forall x \in\right] 0, L_{1}[, \\
W_{y}^{1}(0, t)=0, I_{1} \sigma_{x}^{1}(0, t)=0, \\
W_{y}^{1}\left(L_{1}, t\right)=0, \frac{\partial \tilde{W}_{y}^{1}}{\partial x}\left(L_{1}, t\right)=0 .
\end{gathered}
$$

Two methods can be used to obtain the natural frequencies and the stress mode shapes:

- by analogy between the stress eigenvalue problem and an equivalent displacement eigenvalue problem.

To obtain the stress problem, one should combine time second derivation of (A2) with equation (A1):

$$
\begin{gathered}
\left.\rho_{1} S_{1} \frac{\partial \sigma_{x}^{1}}{\partial t^{2}}(x, t)+E_{1} I_{1} \frac{\partial^{4} \sigma_{x}^{1}}{\partial x^{4}}(x, t)=0, \quad \forall x \in\right] 0, L_{1}[ \\
I_{1} \frac{\partial^{2} \sigma_{x}^{1}}{\partial x^{2}}(0, t)=0, \quad I_{1} \sigma_{x}^{1}(0, t)=0, \quad I_{1} \frac{\partial^{2} \sigma_{x}^{1}}{\partial x^{2}}\left(L_{1}, t\right)=0, \quad I_{1} \frac{\partial^{3} \sigma_{x}^{1}}{\partial x^{3}}\left(L_{1}, t\right)=0 .
\end{gathered}
$$

After a separation of time and space,

$$
\sigma_{x}^{1 p}(x, t)=\tilde{\sigma}_{x}^{1 p}(x) e^{j \omega_{p}^{1} . t}
$$

the stress eigenvalue problem can be written:

$$
\begin{gathered}
\left.-\rho_{1} S_{1}\left(\omega_{p}^{1}\right) \tilde{\sigma}_{x}^{1 p}(x)+E_{1} I_{1} \frac{\partial^{4} \tilde{\sigma}_{x}^{1 p}}{\partial x^{4}}(x)=0, \quad \forall x \in\right] 0, L_{1}[ \\
I_{1} \frac{\partial^{2} \tilde{\sigma}_{x}^{1 p}}{\partial x^{2}}(0)=0, \quad I_{1} \tilde{\sigma}_{x}^{1 p}(0)=0, ; \quad I_{1} \frac{\partial^{2} \tilde{\sigma}_{x}^{1 p}}{\partial x^{2}}\left(L_{1}\right)=0, \quad I_{1} \frac{\partial^{3} \tilde{\sigma}_{x}^{1 p}}{\partial x^{3}}\left(L_{1}\right)=0 .
\end{gathered}
$$


This problem is analogous to classical displacement eigenvalue problem of a beam simply supported in $x=0$ and free in $x=L_{1}$. Then, the solutions can be expressed by $\left(\omega_{p}^{1} \neq 0\right)$ :

$$
\left\{\begin{array}{l}
\bullet \omega_{p}^{1}=\sqrt{\frac{E_{1} I_{1}}{\rho_{1} S_{1}}}\left(k_{p}^{1}\right)^{2} \quad \text { with } k_{p}^{1} / \tan \left(k_{p}^{1} L_{1}\right)=\tanh \left(k_{p}^{1} L_{1}\right), \\
\bullet \int_{0}^{L_{1}} \rho_{1} S_{1}\left(\tilde{\sigma}_{x}^{1 p}(x)\right)^{2} d x=\rho_{1} S_{1} L_{1}, \\
\bullet \tilde{\sigma}_{x}^{1 p}(x)=\cosh \left(k_{p}^{1}\left(L_{1}-x\right)\right)+\cos \left(k_{p}^{1}\left(L_{1}-x\right)\right) \\
-\frac{\cosh \left(k_{p}^{1} L_{1}\right)-\cos \left(k_{p}^{1} L_{1}\right)}{\sinh \left(k_{p}^{1} L_{1}\right)-\sin \left(k_{p}^{1} L_{1}\right)} \cdot\left(\sinh \left(k_{p}^{1}\left(L_{1}-x\right)\right)+\sin \left(k_{p}^{1}\left(L_{1}-x\right)\right)\right)
\end{array}\right.
$$

As the Helmholtz mode for an acoustic cavity problem, the beam simply supported - clamped has one stress mode of null natural frequency (see section 3.4.2 of part I). Indeed by analogy with displacement equation of motion, this mode corresponds to the rigid body mode of the beam simply supported - free. It is expressed by:

$$
\left\{\begin{array}{l}
\omega_{0}^{1}=0 \\
\tilde{\sigma}_{x}^{1,0}(x)=x
\end{array}\right.
$$

It can be noticed that this method of calculation of stress mode shapes can be used only for simple structure where the analogy with displacement equations is possible. This method does not permit to obtain directly the generalised mass or the generalised stiffness as defined in section 3 of part I (equations (45-49)).

- by the calculation of the displacement mode shapes of the displacement eigenvalue problem and by using the constitutive low (see reference [9]).

The displacement eigenvalue problem are obtained directly by introducing (A1) in (A2) and by taking into account boundary conditions.

With a separation of time and space $W_{y}^{1}(x, t)=\tilde{W}^{1 p}(x) e^{j \omega_{p}^{1} . t}$, solutions of the problem are finally: 


$$
\left\{\begin{array}{l}
\bullet \omega_{p}^{1}=\sqrt{\frac{E_{1} I_{1}}{\rho_{1} S_{1}}}\left(k_{p}^{1}\right)^{2} \quad \text { with } k_{p}^{1} / \tan \left(k_{p}^{1} L_{1}\right)=\tanh \left(k_{p}^{1} L_{1}\right), \\
\bullet M_{p}^{1}=\rho_{1} S_{1} L_{1}, \\
\bullet \tilde{W}_{y}^{1 p}(x)=\cosh \left(k_{p}^{1}\left(L_{1}-x\right)\right)-\cos \left(k_{p}^{1}\left(L_{1}-x\right)\right) \\
\quad-\frac{\cosh \left(k_{p}^{1} L_{1}\right)-\cos \left(k_{p}^{1} L_{1}\right)}{\sinh \left(k_{p}^{1} L_{1}\right)-\sin \left(k_{p}^{1} L_{1}\right)} \cdot\left(\sinh \left(k_{p}^{1}\left(L_{1}-x\right)\right)-\sin \left(k_{p}^{1}\left(L_{1}-x\right)\right)\right),
\end{array}\right.
$$

Using the constitutive law (A2), one deduces the stress shape associated to the $p^{\text {th }}$ mode:

$$
\begin{aligned}
\tilde{\sigma}_{x}^{1 p}(x)=\left(k_{p}^{1}\right)^{2} E_{1} I_{1}[ & \cosh \left(k_{p}^{1}\left(L_{1}-x\right)\right)+\cos \left(k_{p}^{1}\left(L_{1}-x\right)\right) \\
& \left.-\frac{\cosh \left(k_{p}^{1} L_{1}\right)-\cos \left(k_{p}^{1} L_{1}\right)}{\sinh \left(k_{p}^{1} L_{1}\right)-\sin \left(k_{p}^{1} L_{1}\right)} \cdot\left(\sinh \left(k_{p}^{1}\left(L_{1}-x\right)\right)+\sin \left(k_{p}^{1}\left(L_{1}-x\right)\right)\right)\right] .
\end{aligned}
$$

By this calculation one cannot determinate the stress mode of null frequency. However, for the CLF determination it is not important because this mode is always non resonant and thus it is not necessary to take it into account. 


\section{APPENDIX B: NOMENCLATURE}

$A_{1}, A_{2}$

$b_{1}, b_{2}$

$B_{1}, B_{2}$

$E_{1}, E_{2}$

$<E_{t}^{1}>,<E_{t}^{2}>$

$f_{k}^{1 i}, f_{k}^{2 i}$

$\tilde{f}_{p k}^{1 i}, \tilde{f}_{q k}^{2 i}$

$h_{1}, h_{2}$

$H_{1}, H_{2}$

$I_{1}, I_{2}$

$k_{p}^{1}, k_{q}^{2}$

$K_{p}^{1}, K_{q}^{2}$

$L_{1}, L_{2}$

$\tilde{\mathbf{M}}_{f}^{1 p}$

$M_{p}^{1}, M_{q}^{2}$

$n_{1}, n_{2}$

$N_{1}, N_{2}$

$N_{1}^{1}, N_{1}^{2}$

$N_{2}^{1}, N_{2}^{2}$

$S_{1}, S_{2}$

$u_{k}^{1 i}, u_{k}^{2 i}$

$\tilde{u}_{p k}^{1 i}, \tilde{u}_{q k}^{2 i}$ plate length of the common edge

beam width

plate length

Young modulus

averaged total energy obtained from numerical experiment

force nodal variable

force nodal mode shape

beam thickness

plate thickness

beam second moment area

modal wave number

modal stiffness

beam length

flexural moment mode shape of beam 1

modal mass

modal density

number of resonant modes in the considered frequency bandwidth

modal order of resonant mode with the lowest natural frequency in $\Delta \omega$

modal order of resonant mode with the highest natural frequency in $\Delta \omega$

beam cross section

displacement nodal variable

displacement nodal mode shape 
$\mathbf{W}_{p q}^{12}$

$W_{i}^{1}, W_{i}^{2}$

$W_{y}^{1}, W_{y}^{2}$

$\tilde{W}_{y}^{1 p}, \tilde{W}_{y}^{2 q}$

$\beta_{p q}^{12}$

$\Delta \omega$

$\Delta_{p}^{1}, \Delta_{q}^{2}$

$\eta_{12}$

$\eta_{12}^{n e}$

$\gamma_{p q}^{12}$

$v_{1}, v_{2}$

$\tilde{\theta}_{z}^{2 q}$

$\omega_{c}$

$\omega_{p}^{1}, \omega_{q}^{2}$

$\rho_{1}, \rho_{2}$

$\sigma_{i j}^{1}, \sigma_{i j}^{2}$

$\sigma_{x}^{1}, \sigma_{x}^{2}$

$\tilde{\sigma}_{x}^{1 p}$ interaction modal work between mode $p$ of subsystem 1 and mode $q$ of

subsystem 2

displacement vector

beam generalised transversal displacement

generalised displacement mode shape

Intermodal Coupling Factor (ICF) between mode $p$ of subsystem 1 and mode $q$ of subsystem 2

angular frequency bandwidth of interest

modal damping bandwidth

Coupling Loss Factor (CLF)

CLF obtained by SEA matrix - inversion technique with numerical

experiment

Damping Loss Factor (DLF)

gyroscopic modal coupling coefficient between mode $p$ of subsystem 1 and mode $q$ of subsystem 2

Poisson's coefficient

angular rotation mode shape of beam 2

central angular radian frequency of the frequency bandwidth $\Delta \omega$

mode's natural angular frequency

mass density

stress tensor

beam generalised normal stress

generalised stress mode shape of beam 1 


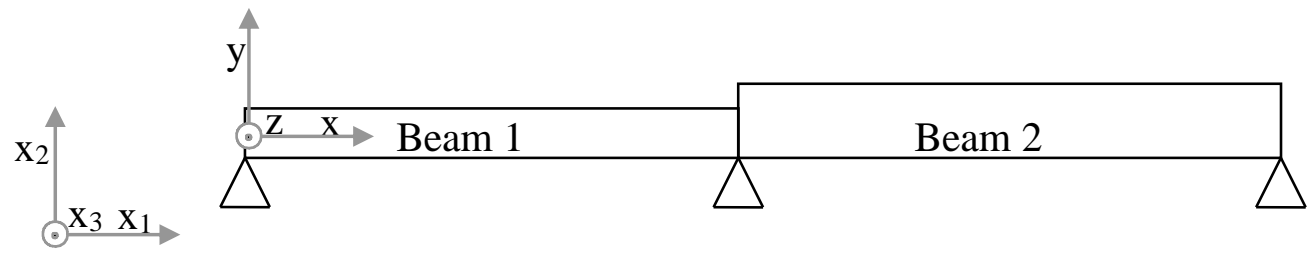

Figure 1: Two pinned-pinned beams coupled rigidly at one end. 


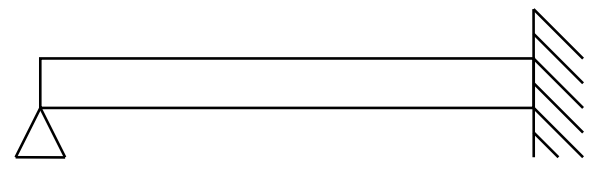

(a)

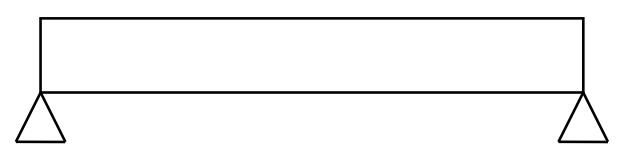

(b)

Figure 2: Uncoupled beams (a) Uncoupled - blocked subsystem, beam 1 (b) Uncoupled - free subsystem, beam 2 . 
FIGURE 3

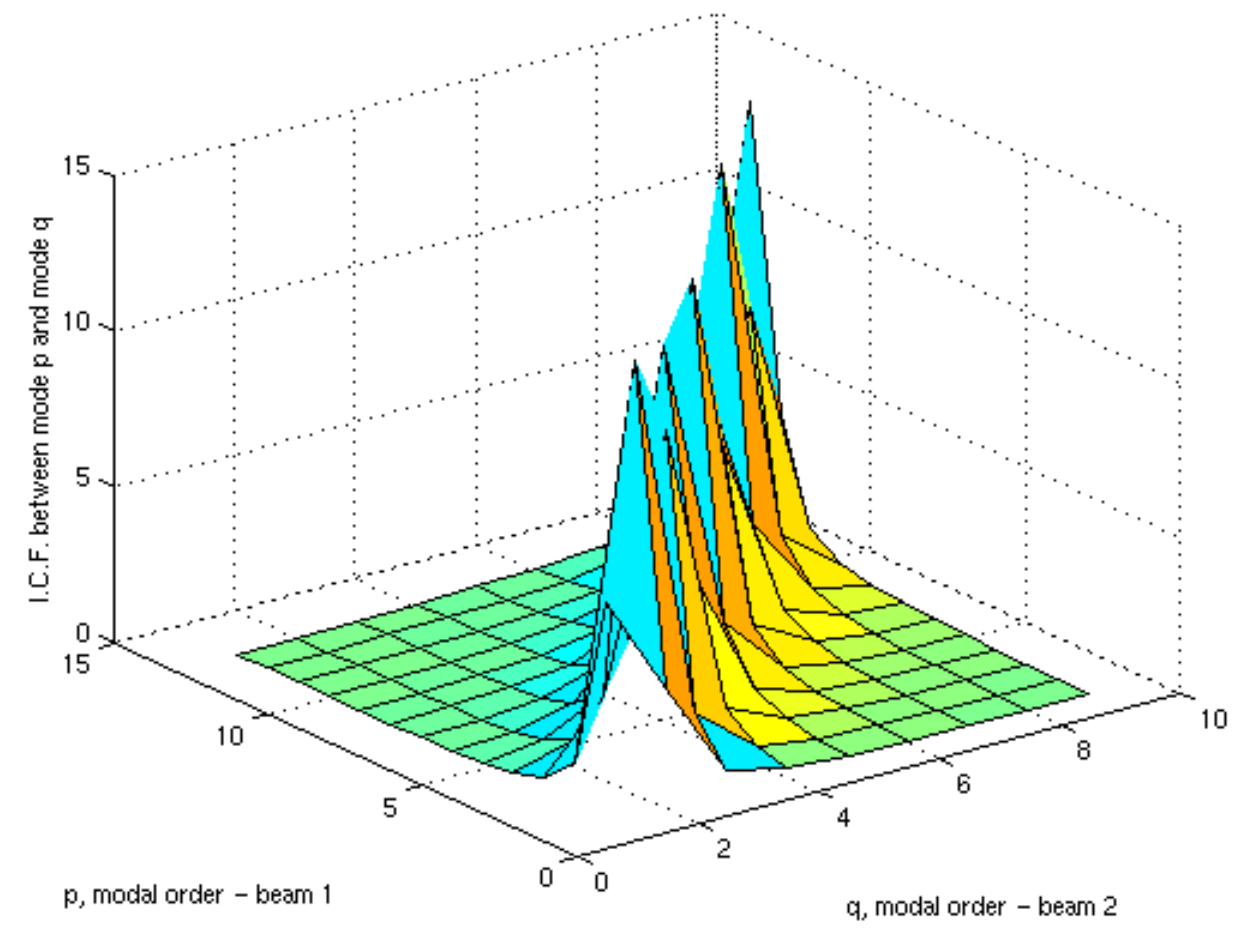

Figure 3: Intermodal Coupling Factors versus couples of resonant modes of the two beams, third octave band $16000 \mathrm{~Hz} ; L_{1}=2.5 \mathrm{~m}, L_{2}=3.5 \mathrm{~m}, b_{1}=b_{2}=0.01 \mathrm{~m}, h_{1}=0.001 \mathrm{~m}, h_{2}=0.004 \mathrm{~m}, \eta_{1}=\eta_{2}=0.01$. 


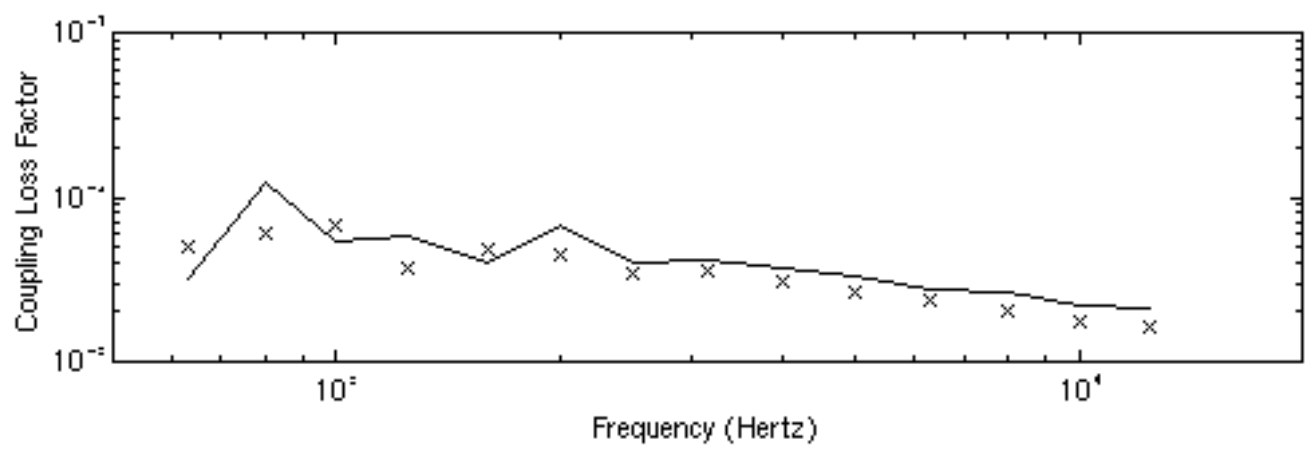

(a)

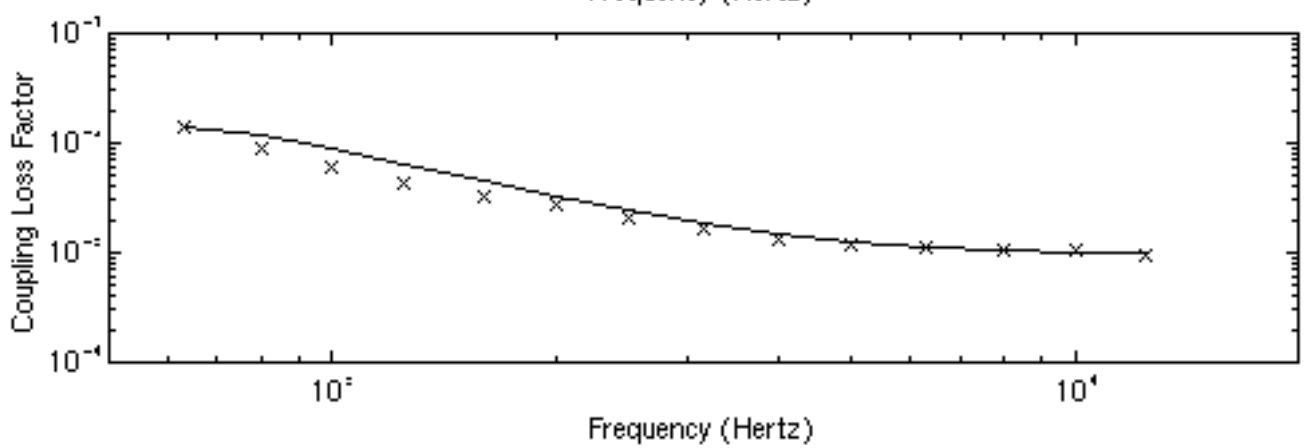

(b)

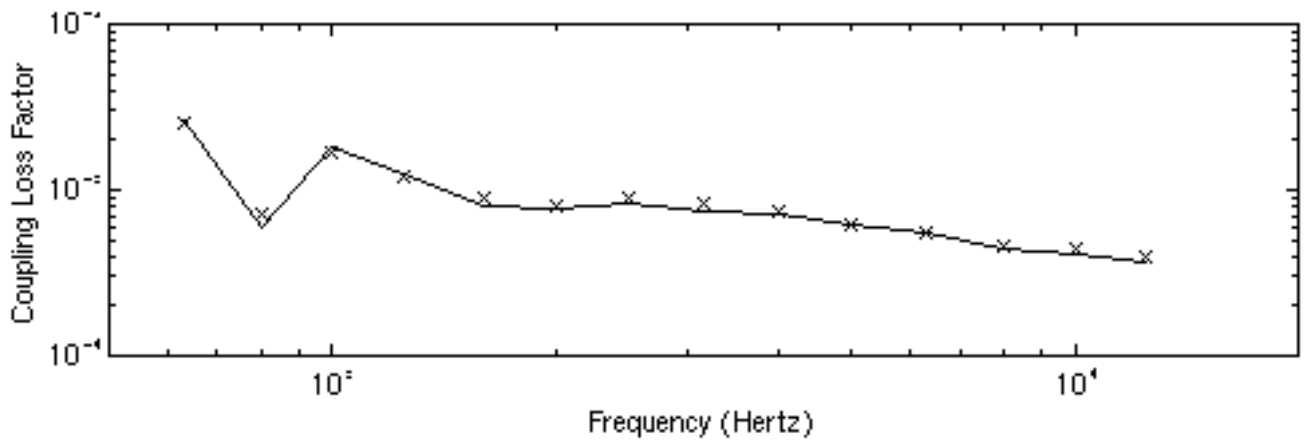

(c)

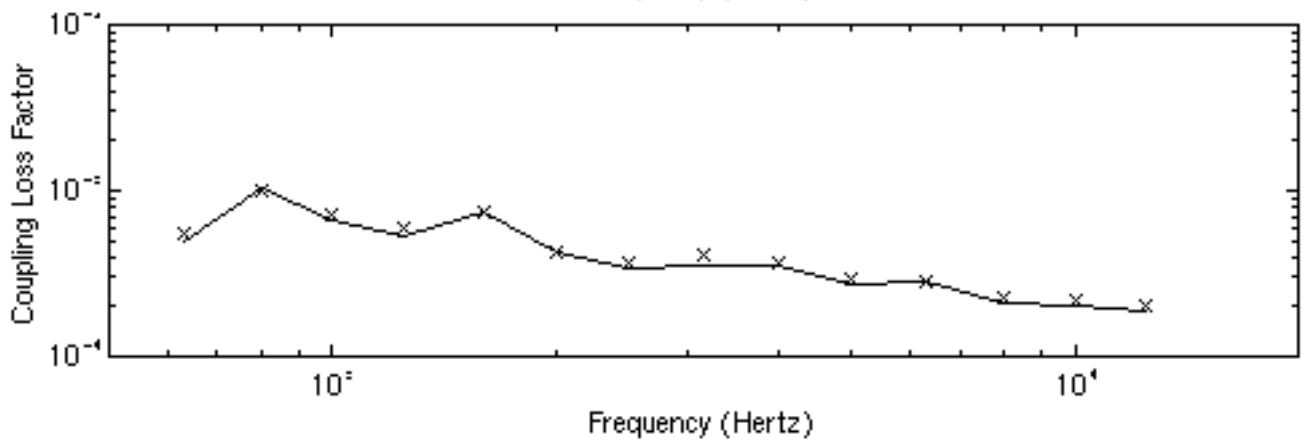

(d)

Figure 4: $\quad$ Coupling Loss Factor versus frequency; One-third octave band results; (a) $h_{2} / h_{1}=1.5$, (b) $h_{2} / h_{1}=2$, (c) $h_{2} / h_{1}=3$, (d) $h_{2} / h_{1}=4 ; L_{1}=2.5 \mathrm{~m}, L_{2}=3.5 \mathrm{~m}, b_{1}=b_{2}=0.01 \mathrm{~m}, h_{1}=0.001 \mathrm{~m}, \eta_{1}=\eta_{2}=0.01$; Two calculations: ——, present approach; x, SEA matrix -inversion. 


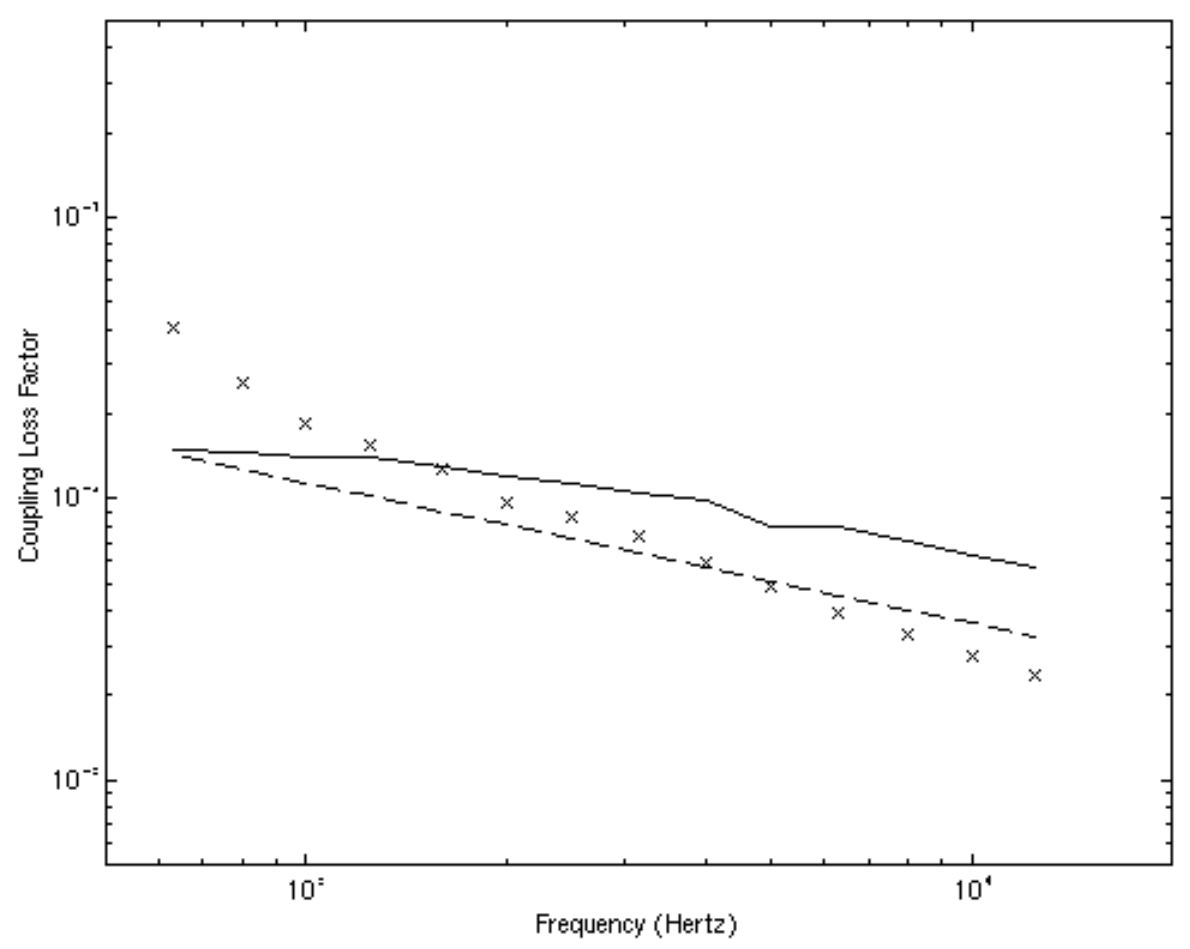

Figure 5: Coupling Loss Factor versus frequency; One third-octave band results; $L_{1}=L_{2}=2.5 \mathrm{~m}$, $b_{1}=b_{2}=0.01 \mathrm{~m}, h_{1}=h_{2}=0.001 \mathrm{~m}, \eta_{1}=\eta_{2}=0.01$. Three calculations: - - present approach; $\mathrm{x}$, SEA matrix -inversion; ----, travelling wave approach. 


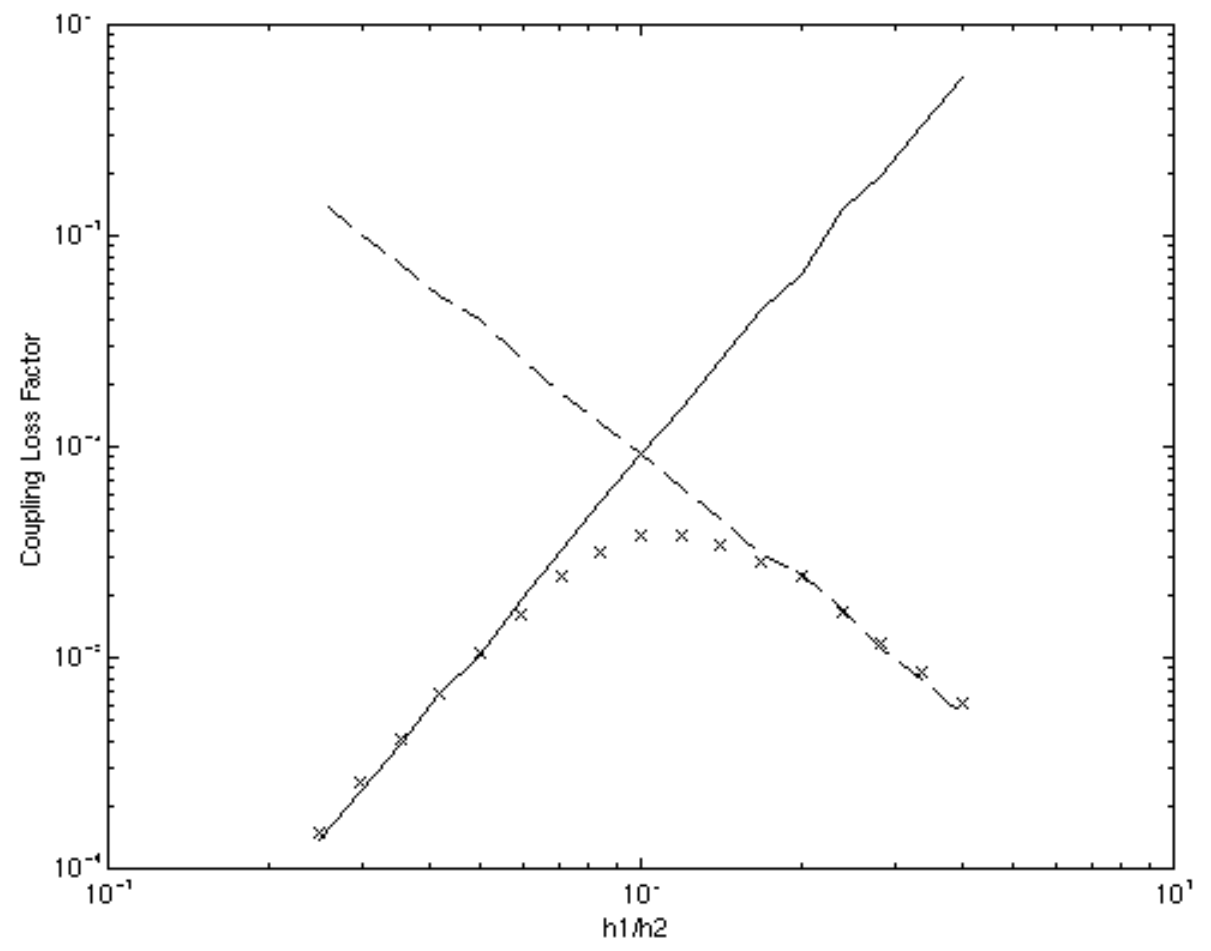

Figure 6: Coupling Loss Factor versus thickness ratio $h_{1} / h_{2}$; Third-octave band centred on 10000 $\mathrm{Hz} ; L_{1}=2.5 \mathrm{~m}, L_{2}=3.5 \mathrm{~m}, b_{1}=b_{2}=0.01 \mathrm{~m}, h_{1}=0.001 \mathrm{~m}, \eta_{1}=\eta_{2}=0.01$; Three calculations: - , present approach with blocked modes for beam 1 and free modes for beam 2; ----, present approach with free modes for beam 1 and blocked modes for beam 2; x, SEA matrix -inversion. 


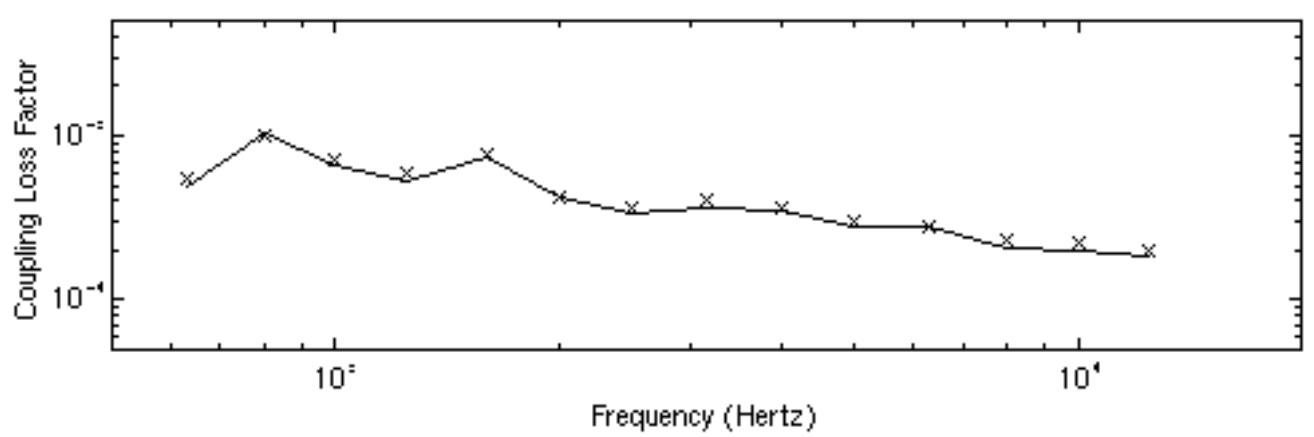

(a)

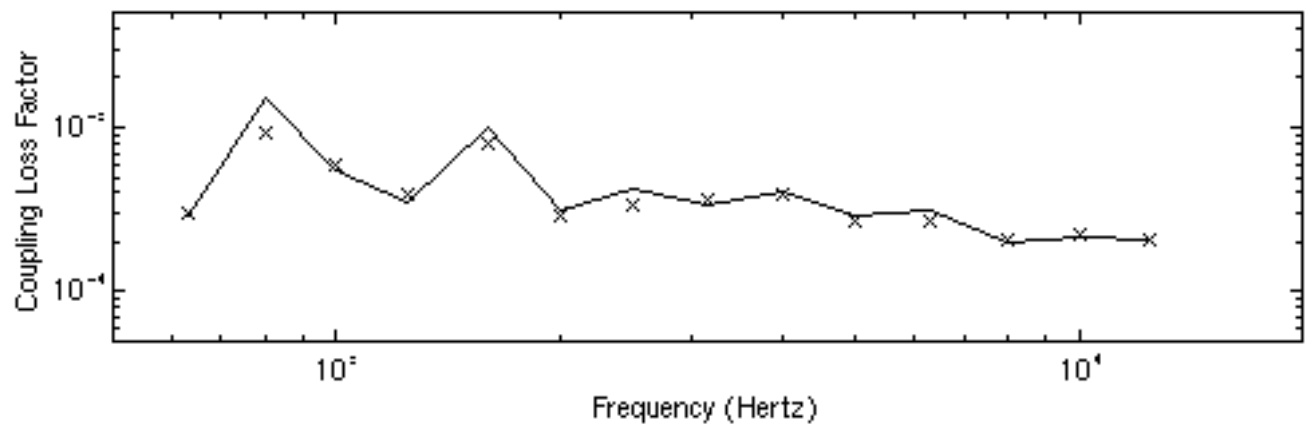

(b)

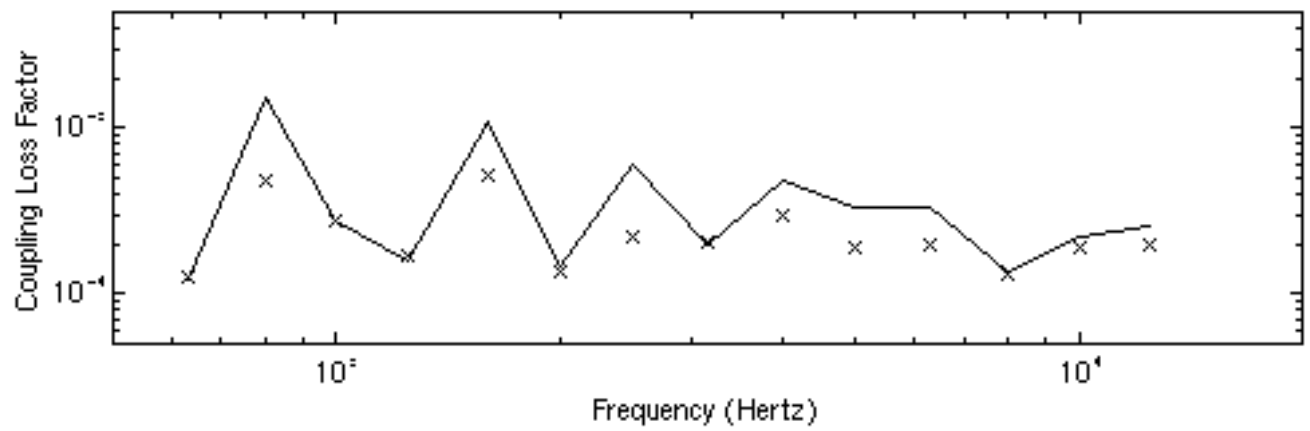

(c)

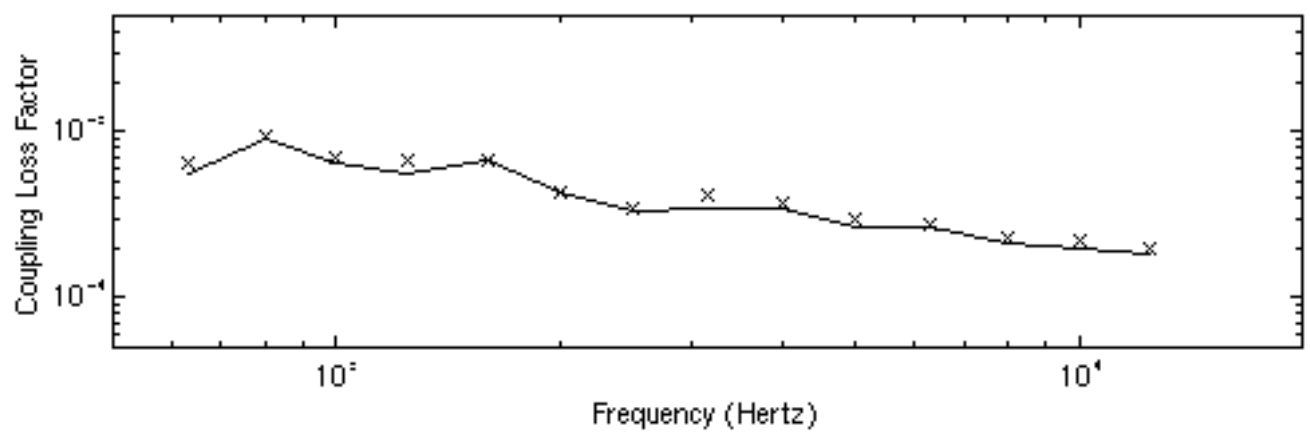

(d)

Figure 7: Coupling Loss Factor versus frequency; One third-octave band results; (a) $\eta_{I}=\eta_{2}=0.01$, (b) $\eta_{1}=\eta_{2}=0.005$, (c) $\eta_{1}=\eta_{2}=0.002$, (d) $\eta_{1}=0.02, \eta_{2}=0.005 ; L_{1}=2.5 \mathrm{~m}, L_{2}=3.5 \mathrm{~m}, b_{1}=b_{2}=0.01 \mathrm{~m}$, $h_{l}=0.001 \mathrm{~m}, h_{2}=0.004 \mathrm{~m}$; Two calculations: - 


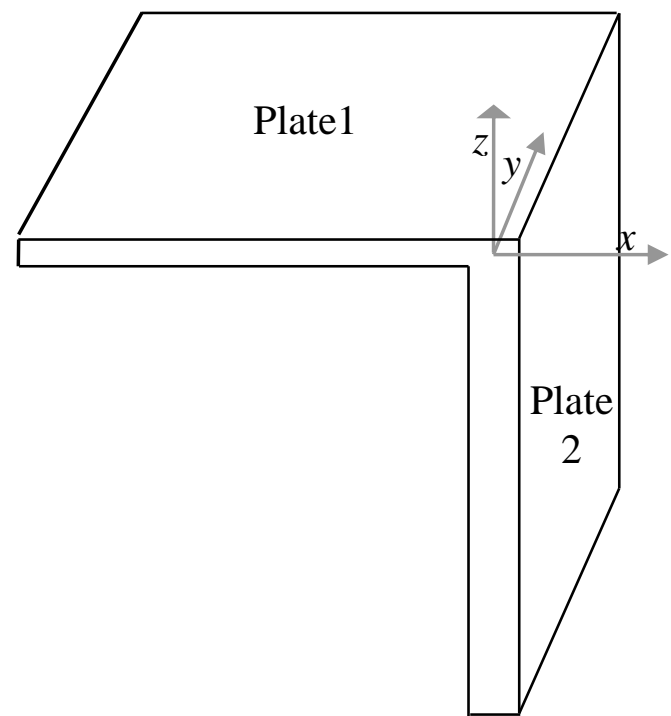

Figure 8: Illustration of an L-shaped plate. 


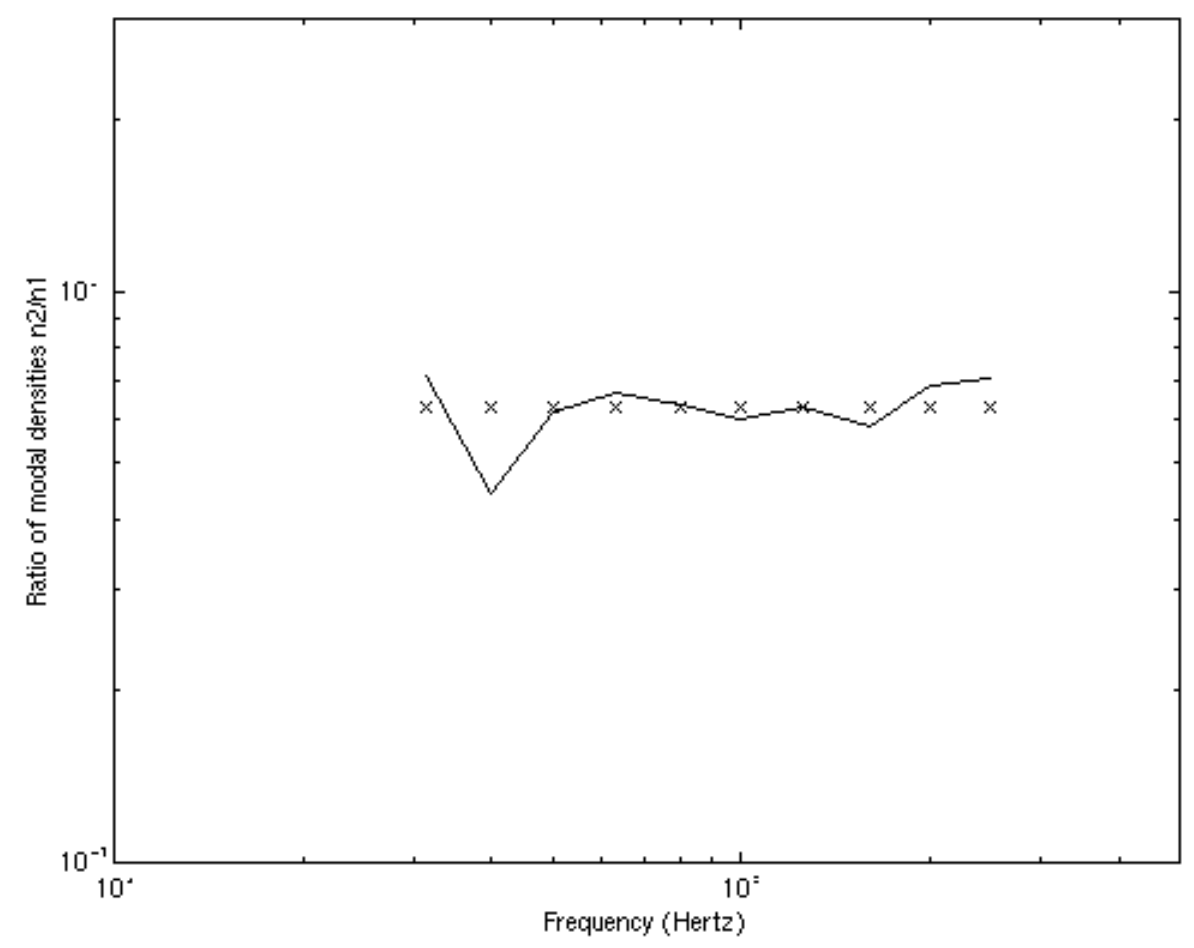

Figure 9: Modal densities ratio versus frequency, third octave band calculation. - - FEM result; x, with expression (20). 


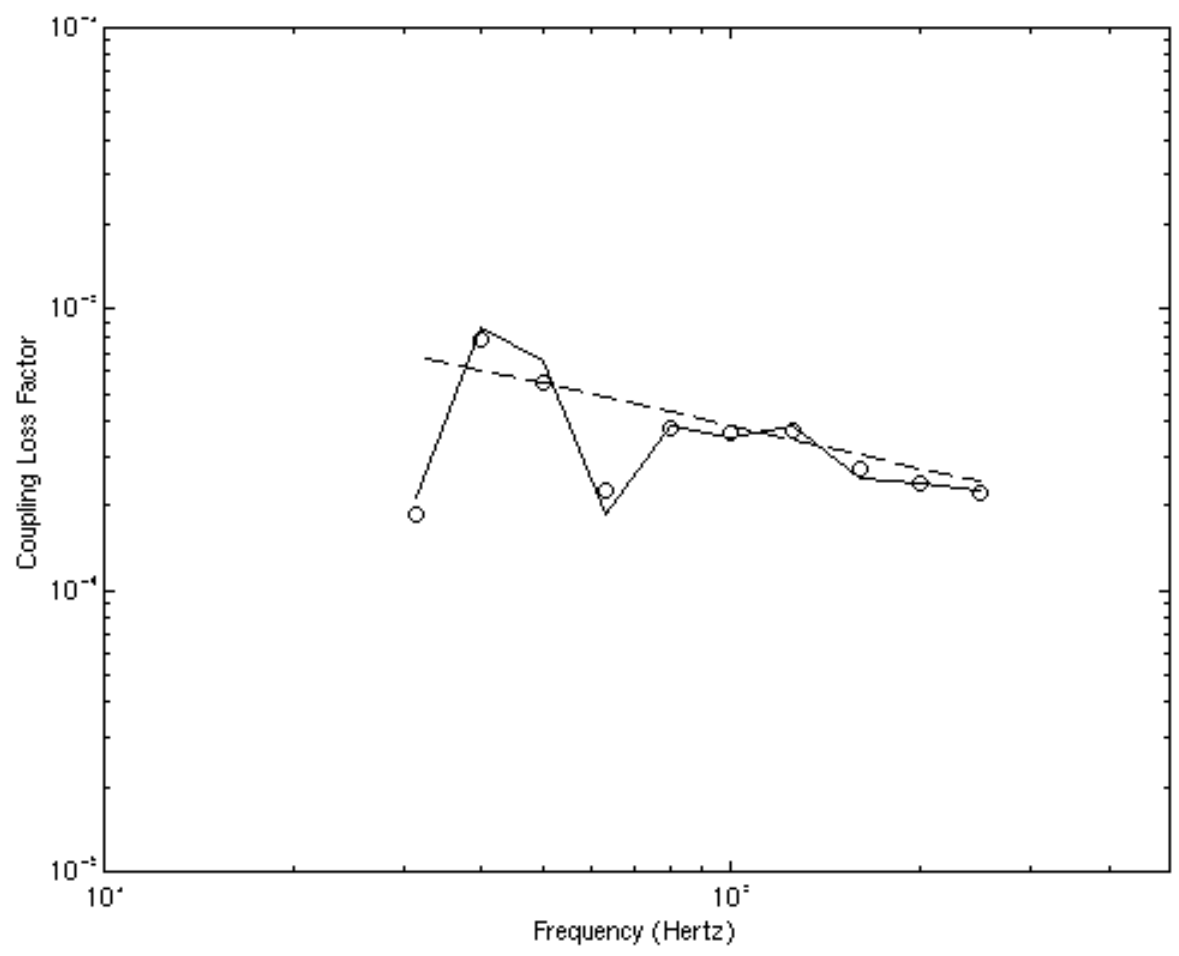

Figure 10: Coupling Loss Factor versus frequency, third octave band calculation; Three calculations: - , CLF-DMF/FEM; o, SEA matrix -inversion; ----, travelling wave approach. 


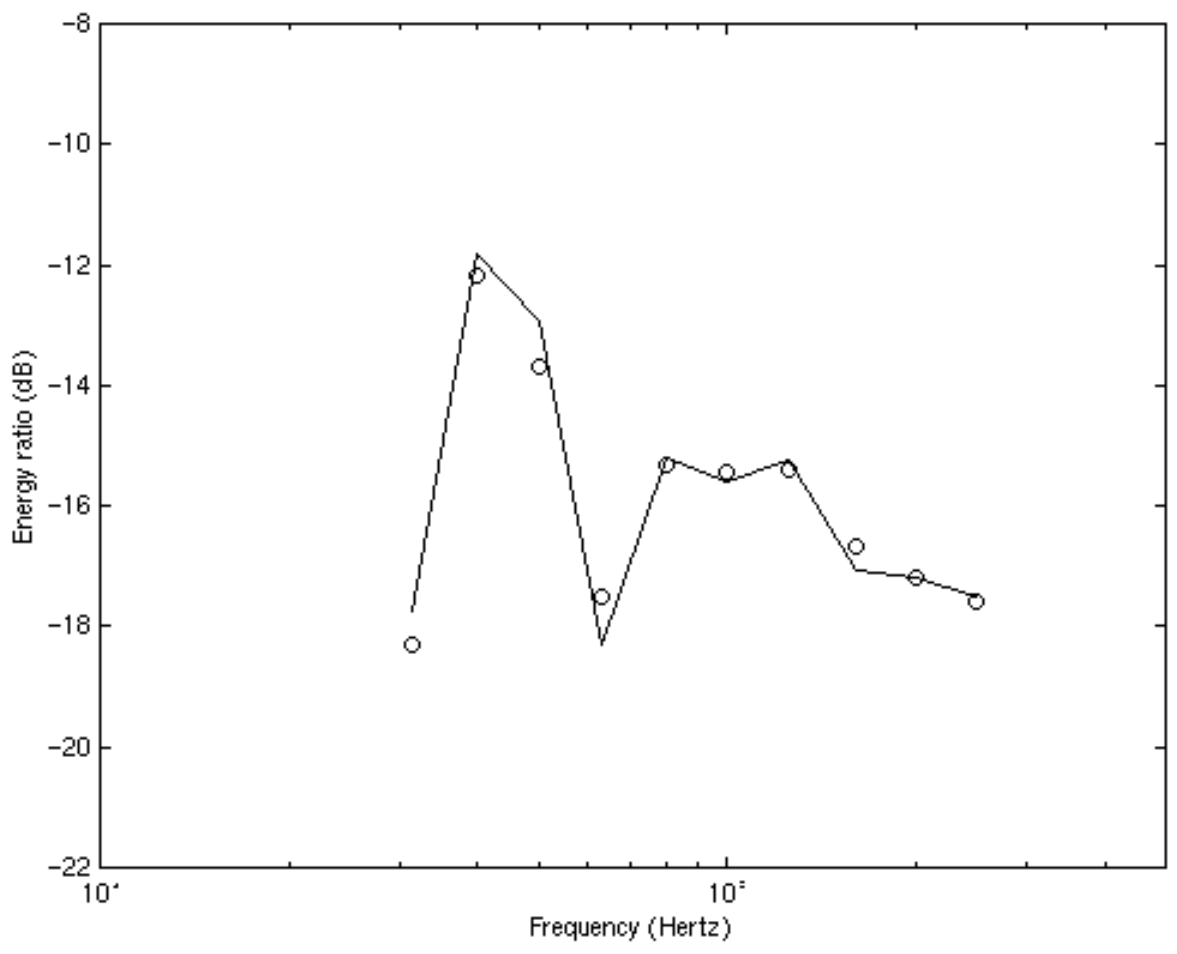

Figure 11: Energy ratio E1/E2 versus frequency, third octave band calculation, plate 2 excited; Two calculations: — - SEA result with CLF obtained by DMF/FEM; o, exact result. 


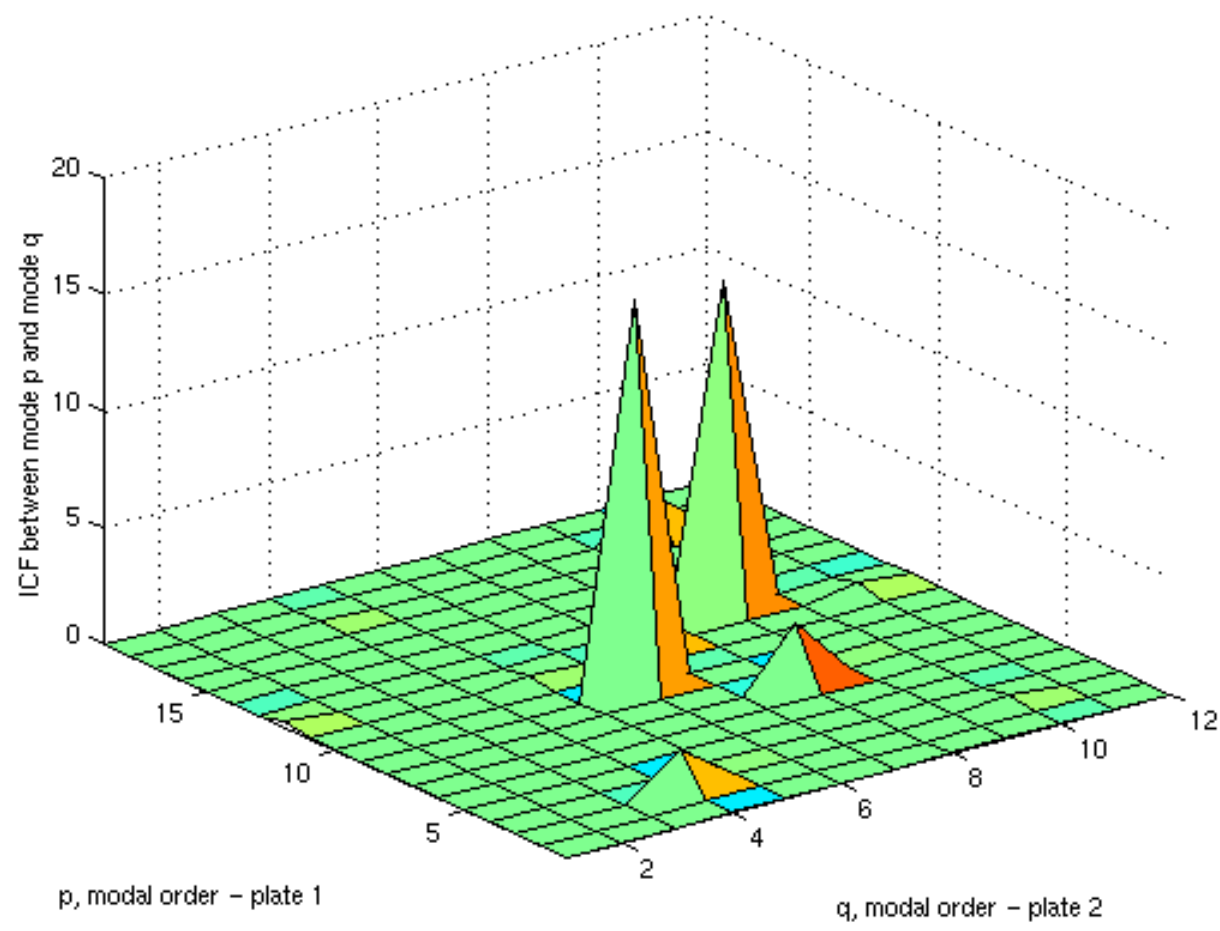

Figure 12: Intermodal Coupling Factors versus couples of resonant modes of the two plates, third octave band $800 \mathrm{~Hz}$; DMF/FEM results; Modes classified with increasing natural frequencies 


\begin{tabular}{c|cc}
\hline & Plate 1 & Plate 2 \\
\hline Length of the common edge & $A_{l}=1 \mathrm{~m}$ & $A_{2}=1 \mathrm{~m}$ \\
Other length & $B_{l}=1 \mathrm{~m}$ & $B_{2}=2.5 \mathrm{~m}$ \\
Thickness & $H_{l}=3 \mathrm{~mm}$ & $H_{2}=12 \mathrm{~mm}$ \\
Modulus of elasticity & $E_{l}=2 \times 10^{+11} \mathrm{~Pa}$ & $E_{2}=2 \times 10^{+11} \mathrm{~Pa}$ \\
Density & $\rho_{l}=7800 \mathrm{Kg} / \mathrm{M}^{3}$ & $\rho_{2}=7800 \mathrm{Kg} / \mathrm{M}^{3}$ \\
Poisson ratio & $v_{l}=0.3$ & $v_{2}=0.3$ \\
Damping Loss Factor & $\eta_{l}=0.02$ & $\eta_{2}=0.02$ \\
\hline
\end{tabular}

Table 1: Definition and values of parameters. 


\begin{tabular}{|c|c|c|c|}
\hline & $\begin{array}{c}\text { Mode } \\
\text { order }\end{array}$ & Natural frequency & $\begin{array}{c}\text { Mode shape on common edge } \\
i \in\{\text { Coupling node set }\}\end{array}$ \\
\hline Plate 1 & $p$ & $\begin{array}{c}\omega_{p}^{1} / \\
1 \mathrm{~Hz}<\frac{\omega_{p}^{1}}{2 \pi}<3000 H z\end{array}$ & $\begin{array}{c}\text { 'SINGLE POINT CONSTRAINT FORCE' } \\
\text { SPCFORCE } \rightarrow\left\{\tilde{f}_{p 1}^{1 i}, \tilde{f}_{p 2}^{1 i}, \tilde{f}_{p 3}^{1 i}, \tilde{f}_{p 4}^{1 i}, \tilde{f}_{p 5}^{1 i}, \tilde{f}_{p 6}^{1 i}\right\}\end{array}$ \\
\hline Plate 2 & $q$ & $\begin{array}{c}\omega_{q}^{2} / \\
\omega_{q}^{2}\end{array}$ & 'DISPLACEMENT' \\
& & & DISPLACEMENT $\rightarrow\left\{\tilde{u}_{q 1}^{2 i}, \tilde{u}_{q 2}^{2 i}, \tilde{u}_{q 3}^{2 i}, \tilde{u}_{q 4}^{2 i}, \tilde{u}_{q 5}^{2 i}, \tilde{u}_{q 6}^{2 i}\right\}$ \\
\hline
\end{tabular}

Table 2: Definition of the extracted FEM output data for each plate. $i$ : node on the common edge (coupling node set). Generalized masses normalised to one. 
TABLE 3

L.MAXIT, J.L. GUYADER

\begin{tabular}{l|cccccccccc}
\hline & $315 \mathrm{~Hz}$ & $400 \mathrm{~Hz}$ & $500 \mathrm{~Hz}$ & $630 \mathrm{~Hz}$ & $800 \mathrm{~Hz}$ & $1000 \mathrm{~Hz}$ & $1250 \mathrm{~Hz}$ & $1600 \mathrm{~Hz}$ & $2000 \mathrm{~Hz}$ & $2500 \mathrm{~Hz}$ \\
\hline plate 1 & 7 & 9 & 13 & 12 & 19 & 25 & 27 & 38 & 47 & 57 \\
plate 2 & 5 & 4 & 8 & 8 & 12 & 15 & 17 & 22 & 32 & 40 \\
\hline
\end{tabular}

Table 3: Number of modes per band for each plate. Finite element results. 